\title{
Measurement of $Z$ Decays into Lepton Pairs
}

G. S. Abrams, (1) C. E. Adolphsen, ${ }^{(2)}$ D. Averill, ${ }^{(3)}$ J. Ballam, ${ }^{(4)}$ B. C. Barish, ${ }^{(5)}$ T. Barklow, ${ }^{(4)}$ B. A. Barnett, ${ }^{(6)}$ J. Bartelt, ${ }^{(4)}$ S. Bethke, ${ }^{(1)}$ D. Blockus, ${ }^{(3)}$ G. Bonvicini, ${ }^{(7)}$ A. Boyarski, ${ }^{(4)}$ B. Brabson, ${ }^{(3)}$ A. Breakstone, ${ }^{(8)}$ F. Bulos, ${ }^{(4)}$ P. R. Burchat, ${ }^{(2)}$ D. L. Burke, ${ }^{(4)}$ R. J. Cence, ${ }^{(8)}$ J. Chapman, ${ }^{(7)}$ M. Chmeissani, ${ }^{(7)}$ D. Cords, ${ }^{(4)}$ D. P. Coupal, ${ }^{(4)}$ P. Dauncey, ${ }^{(6)}$ H. C. DeStaebler, ${ }^{(4)}$ D. E. Dorfan, ${ }^{(2)}$ J. M. Dorfan, ${ }^{(4)}$ D. C. Drewer, ${ }^{(6)}$ R. Elia, ${ }^{(4)}$ G. J. Feldman, ${ }^{(4)}$ D. Fernandes, ${ }^{(4)}$ R. C. Field, ${ }^{(4)}$ W. T. Ford, ${ }^{(9)}$ C. Fordham, ${ }^{(4)}$ R. Frey, ${ }^{(7)}$ D. Fujino, ${ }^{(4)}$ K. K. Gan, ${ }^{(4)}$ E. Gero, ${ }^{(7)}$ G. Gidal, ${ }^{(1)}$ T. Glanzman, ${ }^{(4)}$ G. Goldhaber, ${ }^{(1)}$ J. J. Gomez Cadenas, ${ }^{(2)}$ G. Gratta, ${ }^{(2)}$ G. Grindhammer, ${ }^{(4)}$ P. Grosse-Wiesmann, ${ }^{(4)}$ G. Hanson, ${ }^{(4)}$ R. Harr, ${ }^{(1)}$ B. Harral, ${ }^{(6)}$ F. A. Harris, ${ }^{(8)}$ C. M. Hawkes, ${ }^{(5)}$ K. Hayes, ${ }^{(4)}$ C. Hearty, ${ }^{(1)}$ C. A. Heusch, ${ }^{(2)}$ M. D. Hildreth, ${ }^{(4)}$ T. Himel, ${ }^{(4)}$ D. A. Hinshaw, ${ }^{(9)}$ S. J. Hong, ${ }^{(7)}$ D. Hutchinson, ${ }^{(4)}$ J. Hylen, ${ }^{(6)}$ W. R. Innes, ${ }^{(4)}$ R. G. Jacobsen, ${ }^{(4)}$ J. A. Jaros, ${ }^{(4)}$ C. K. Jung, ${ }^{(4)}$ J. A. Kadyk, ${ }^{(1)}$ J. Kent, ${ }^{(2)}$ M. King, ${ }^{(2)}$ S. R. Klein, ${ }^{(4)}$ D. S. Koetke, ${ }^{(4)}$ S. Komamiya, ${ }^{(4)}$ W. Koska, ${ }^{(7)}$ L. A. Kowalski, ${ }^{(4)}$ W. Kozanecki, ${ }^{(4)}$ J. F. Kral, ${ }^{(1)}$ M. Kuhlen, ${ }^{(5)}$ L. Labarga, ${ }^{(2)}$ A. J. Lankford, ${ }^{(4)}$ R. R. Larsen, ${ }^{(4)}$ F. Le Diberder, ${ }^{(4)}$ M. E. Levi, ${ }^{(1)}$ A. M. Litke, ${ }^{(2)}$ X. C. Lou, ${ }^{(3)}$ V. Lüth, ${ }^{(4)}$ J. A. McKenna, ${ }^{(5)}$ J. A. J. Matthews, ${ }^{(6)}$ T. Mattison, ${ }^{(4)}$ B. D. Milliken, ${ }^{(5)}$ K. C. Moffeit, ${ }^{(4)}$ C. T. Munger, ${ }^{(4)}$ W. N. Murray, ${ }^{(3)}$ J. Nash, ${ }^{(4)}$ H. Ogren, ${ }^{(3)}$ K. F. O'Shaughnessy, ${ }^{(4)}$ S. I. Parker, ${ }^{(8)}$ C. Peck, ${ }^{(5)}$ M. L. Perl, ${ }^{(4)}$ F. Perrier, ${ }^{(4)}$ M. Petradza, ${ }^{(4)}$ R. Pitthan, ${ }^{(4)}$ F. C. Porter, ${ }^{(5)}$ P. Rankin, ${ }^{(9)}$ K. Riles, ${ }^{(4)}$ F. R. Rouse, ${ }^{(4)}$ D. R. Rust, ${ }^{(3)}$ H. F. W. Sadrozinski, ${ }^{(2)}$ M. W. Schaad, (1) B. A. Schumm, ${ }^{(1)}$ A. Seiden, ${ }^{(2)}$ J. G. Smith, ${ }^{(9)}$ A. Snyder, ${ }^{(3)}$ E. Soderstrom, ${ }^{(5)}$ D. P. Stoker, ${ }^{(6)}$ R. Stroynowski, ${ }^{(5)}$ M. Swartz, ${ }^{(4)}$ R. Thun, ${ }^{(7)}$ G. H. Trilling, ${ }^{(1)}$ R. Van Kooten, ${ }^{(4)}$ P. Voruganti, ${ }^{(4)}$ S. R. Wagner, ${ }^{(4)}$ S. Watson, ${ }^{(2)}$ P. Weber, ${ }^{(9)}$ A. Weigend, ${ }^{(4)}$ A. J. Weinstein, ${ }^{(5)}$ A. J. Weir, ${ }^{(5)}$ E. Wicklund, ${ }^{(5)}$ M. Woods, ${ }^{(4)}$ D. Y. Wu, ${ }^{(5)}$ M. Yurko, ${ }^{(3)}$ C. Zaccardelli, ${ }^{(2)}$ and C. von Zanthier ${ }^{(2)}$

${ }^{(1)}$ Lawrence Berkeley Laboratory and Department of Physics, University of California, Berkeley, California 94720

${ }^{(2)}$ University of California, Santa Cruz, Santa Cruz, California 95064

${ }^{(3)}$ Indiana University, Bloomington, Indiana 47405

${ }^{(4)}$ Stanford Linear Accelerator Center, Stanford University, Stanford, California 94309

${ }^{(5)}$ California Institute of Technology, Pasadena, California 91125

(6) Johns Hopkins University, Baltimore, Maryland 21218

${ }^{(7)}$ University of Michigan, Ann Arbor, Michigan 48109

${ }^{(8)}$ University of Hawaii, Honolulu, Hawaii 96822

${ }^{(9)}$ University of Colorado, Boulder, Colorado 80309

(Received 24 October 1989)

\begin{abstract}
We present measurements by the Mark II experiment of the ratios of the leptonic partial widths of the $Z$ boson to the hadronic partial width. The results are $\Gamma_{e e} / \Gamma_{\text {had }}=0.037 \pm 8.016, \Gamma_{\mu \mu} / \Gamma_{\text {had }}=0.053 \pm 0.020$, and $\Gamma_{\tau \tau} / \Gamma_{\text {had }}=0.066 \pm 0.021$, in good agreement with the standard-model prediction of 0.048 . From the average leptonic-width result, $\Gamma_{l l} / \Gamma_{\text {had }}=0.053 \pm 8.010$, we derive $\Gamma_{\text {had }}=1.56 \pm 0.28 \mathrm{GeV}$. We find for the vector coupling constants of the tau and muon $v_{\tau}^{2}=0.31 \pm 0.31 \pm 0.43$ and $v_{\mu}^{2}=0.05 \pm 0.30 \pm 0.34$.
\end{abstract}

PACS numbers: $13.38 .+\mathrm{c}, 12.15 . \mathrm{Ji}$

In this Letter we present the first study of $Z$-boson decays into lepton pairs using the Mark II detector at the SLAC Linear Collider. We present the ratio of the partial decay width into lepton pairs to the partial decay width into hadrons,

$$
\frac{\Gamma_{l l}}{\Gamma_{\text {had }}}=\frac{B(Z \rightarrow l l)}{B(Z \rightarrow \text { hadrons })},
$$

which is independent of the center-of-mass energy. In the standard model, this ratio is expected ${ }^{1}$ to be 0.048 .

Data were collected in the $e^{+} e^{-}$center-of-mass energy $\left(E_{\text {c.m. }}\right)$ range of 89.2 to $93.0 \mathrm{GeV}$ and correspond to an integrated luminosity of $19 \mathrm{nb}^{-1}$. A detailed description of the Mark II apparatus can be found in Ref. 2. This analysis uses information mainly from the central drift chamber and the electromagnetic calorimeters. Tracks from charged particles are reconstructed in the drift chamber in the angular range $|\cos \theta|<0.92$, where $\theta$ is the angle with respect to the beam axis. The electromagnetic calorimeters consist of a barrel covering the angular range $|\cos \theta|<0.72$ and end caps extending the coverage to $|\cos \theta|<0.96$. The Mark II dataacquisition system is triggered by either two or more charged tracks within $|\cos \theta|<0.76$, or by an electromagnetic shower with localized energy deposition greater than $3.3 \mathrm{GeV}$ in the barrel or greater than 2.2 $\mathrm{GeV}$ in the end-cap calorimeters.

In order to study the leptonic decays of the $Z$, we select electron-pair, $\mu$-pair, $\tau$-pair, and hadronic-event candidates, and then correct the observed number of events for detection inefficiency and for backgrounds from beam-gas interactions, cosmic rays, two-photon interactions, and misclassified $Z$ decays.

Charged tracks used in the event selection must origi- 
nate from a cylindrical volume of radius $1 \mathrm{~cm}$ and halflength $3 \mathrm{~cm}$ around the beam line, centered at the interaction point. To ensure efficient track reconstruction, the tracks must lie in the angular range $|\cos \theta|<0.82$ and have a transverse momentum with respect to the beam axis greater than $0.15 \mathrm{GeV} / c$. Calorimeter energy depositions greater than $1 \mathrm{GeV}$ that are not associated with a charged track are used together with charged tracks to calculate the visible energy of the event. Events from $Z$ decays are required to have visible energy larger than $15 \%$ of $E_{\text {c.m. }}$ and charged energy larger than $5 \%$ of $E_{\text {c.m. }}$ to minimize contributions from two-photon interactions and from beam-gas interactions.

Hadronic and leptonic decays are separated on the basis of the number of tracks and the event thrust. Hadronic events are selected by requiring at least seven charged tracks coming from the interaction region, and leptonic-event candidates are required to have fewer than seven charged tracks. In addition, lepton candidates must have at least one charged track in each event hemisphere, defined by the plane perpendicular to the thrust axis calculated from the charged particles, and no tracks outside $|\cos \theta|<0.82$.

The separation of the leptonic $Z$ decays into $e, \mu$, and $\tau$ pairs requires additional criteria. Electron events are expected to deposit the full available center-of-mass energy in the calorimeter, and they are separated from $\mu$ and $\tau$ events by requiring that the total calorimeter energy $E_{\text {cal }}$ is larger than $80 \%$ of $E_{\text {c.m. }}$. The missing momentum carried away by neutrinos for $\tau$ events is used to divide the remaining two-prong sample into $\mu$ and $\tau$ candidates. Events are taken as muon candidates if both tracks have momentum larger than $60 \%$ of the beam momentum. Otherwise they are classified as $\tau$ events. The effect of these two cuts is illustrated in Figs. 1 and 2.

In addition, muon events are restricted to the fiducial volume of the barrel calorimeter limited by $|\cos \theta|$ $<0.68$, and the shower energies associated with the

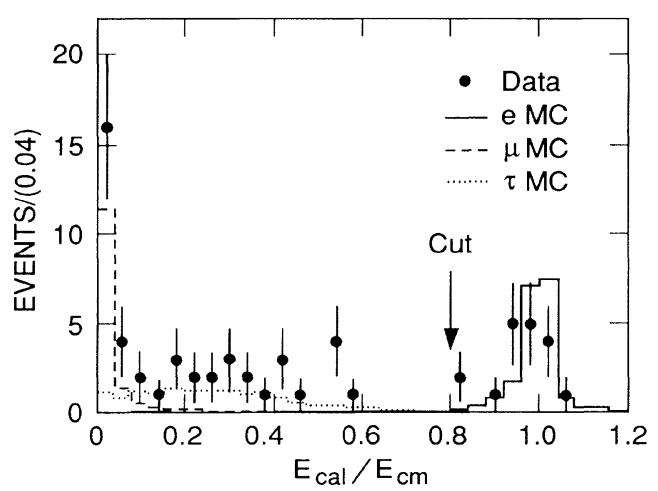

FIG. 1. Calorimeter energy divided by $E_{\text {c.m. for data and }}$ Monte Carlo predictions for electron (solid), muon (dashed), and $\tau$ (dotted) pairs. The arrow indicates the cut at 0.8 to separate electron from muon and $\tau$ events. tracks have to be smaller than $15 \mathrm{GeV}$. Lepton candidates with more than one track in either thrust hemisphere are classified as $\tau$ pairs if the effective mass of the charged tracks in that hemisphere is smaller than 2.2 $\mathrm{GeV} / \mathrm{c}^{2}$ and the event thrust is greater than 0.99 . This cut suppresses background from hadronic events.

The detection efficiencies and contributions from other physics processes are calculated using Monte Carlo simulations. We use the LUND (version 6.3) partonshower model ${ }^{3}$ to simulate hadronic decays of the $Z$. For the $\mu$ - and $\tau$-pair production we use the KORALZ generator, ${ }^{4}$ which includes initial- and final-state radiation effects. Electron-pair production and two-photon interactions are simulated using the generators described in Refs. 5 and 6, respectively. Monte Carlo-generated events are used to simulate raw data events from the Mark II detector. They are combined with data from random beam cross triggers to ensure that the effects of beam-related noise on the reconstruction of events are taken into account. The simulated events are then reconstructed in the same way as the real data.

The detection efficiencies, which include acceptance corrections, for the different classes of events with the selection criteria described above are $88 \%$ for hadronic events, $66 \%$ for $\tau$ pairs, $54 \%$ for $\mu$ pairs. The efficiency for electron pairs produced at the $Z$ peak within $|\cos \theta|$ $<0.82$ is $93 \%$. There are 397 hadronic events, $13 \mu$ pairs, $21 \tau$ pairs, and 18 electron pairs which satisfy the selection criteria.

For the $\tau$ sample the estimated background from $q \bar{q}$ production is 0.6 event, from electron-pair production 0.2 event, and from $\mu$ pairs 0.5 event. For the $\mu$ sample the background from electron and $\tau$ pairs and from hadronic events is less than 0.1 event. The background for the electron sample from all other physics processes is less than 0.15 event. Backgrounds from cosmic rays and

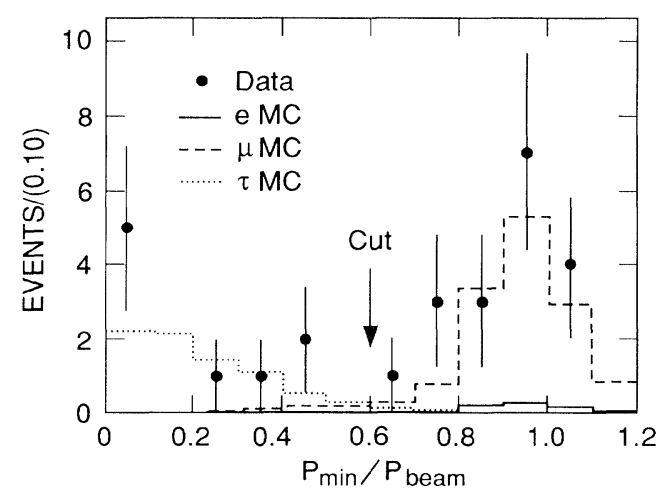

FIG. 2. Ratio of $p_{\text {min }}$ to the beam momentum for two-track events after the cut to remove electron events. $p_{\min }$ is the smaller of the two momenta in the event. The data are shown with a Monte Carlo prediction for electron (solid), muon (dashed), and $\tau$ (dotted) pairs. A cut at 0.6 is used to separate muon and $\tau$ events. 
beam-gas interactions are estimated by displacing the volume which defines the interaction region along the beam direction. We then look for events originating from the displaced volume which otherwise would fulfill the event-selection criteria. From this procedure we estimate a background of 0.15 event for the $\mu$ sample, 0.3 event for the $\tau$ sample, and $<0.4$ event for the hadronic sample. The remaining backgrounds in the $q \bar{q}$ and leptonic samples are negligible.

The electron sample has a sizable contribution from QED and weak-electromagnetic interference. To reduce this contribution we make the additional demand that the positron scattering angle $\theta$ be in the range -0.82 $<\cos \theta<0.68$, eliminating the forward region where the QED contribution is largest. This cut leaves twelve events with a background of $1.4 \pm 0.6$ events due to QED and weak-electromagnetic interference. The overall efficiency after all cuts is $62 \%$.

The systematic errors include the uncertainty of the calorimeter energy scale and the trigger efficiency, the estimate of the effect of beam-related noise and the model dependence of the Monte Carlo generation of hadronic events; in all cases they are much smaller than the statistical errors.

After inclusion of all systematic and statistical uncertainties ${ }^{7}$ the resulting ratios of the partial decay widths $\operatorname{are}^{8}$

$$
\begin{aligned}
& \Gamma_{e e} / \Gamma_{\mathrm{had}}=0.037 \pm 0.012, \\
& \Gamma_{\mu \mu} / \Gamma_{\mathrm{had}}=0.053 \pm 0.015, \\
& \Gamma_{\tau \tau} / \Gamma_{\mathrm{had}}=0.066 \pm 0.0217 .
\end{aligned}
$$

These results are consistent with each other and agree well with the standard-model prediction of 0.048 . Under the assumption of lepton universality the combined lepton sample yields $\Gamma_{l l} / \Gamma_{\text {had }}=0.053 \pm 0.009$.

The hadronic width of the $Z$ depends on the quark couplings and may have additional contributions from new-particle production. Since the lepton couplings are experimentally better constrained than the quark couplings, we assume a standard-model width for the leptons, $\Gamma_{l l}=0.083, \mathrm{GeV}$, and derive from our measurement the hadronic width, $\Gamma_{\text {had }}=1.56 \pm 0.24 \mathrm{GeV}$. The prediction from the standard-model quark couplings is $\Gamma_{\text {had }}$ $=1.73 \mathrm{GeV}$, in agreement with our measurement.

The number of leptonic events can also be used to provide measurements of the leptonic couplings. At the $Z$, the cross section is proportional to $\left(a_{e}^{2}+v_{e}^{2}\right)\left(a_{l}^{2}+v_{l}^{2}\right)$, where $a$ and $v$ denote the axial-vector and vector couplings of the incoming electrons and the final-state leptons. We compare the measured number of $\mu$-pair and $\tau$-pair events with the expectation from our luminosity measurements and the cross section with $v_{l}$ as a parameter. We use the cross-section formula given in Ref. 9 together with our measured values for the $Z$ mass and width, ${ }^{10} M_{Z}=91.14 \pm 0.12 \mathrm{GeV} / c^{2}$ and $\Gamma=2.42 \pm 0.45$
GeV. If we fix the experimentally well-constrained quantities $a_{e}, v_{e}$, and $a_{l}$ to their standard-model values, we find $v_{\tau}^{2}=0.31 \pm 0.31 \pm_{-0.30}^{+0.43}$ and $v_{\mu}^{2}=0.05 \pm 0.30_{-0.23}^{+0.34}$. The first error represents the statistical error, and the second the systematic error, which is entirely dominated by our uncertainty in the $Z$ width. These results can be compared with previous measurements, $v_{\tau}=-1.04$ $\pm 1.25,{ }^{11} v_{\mu}=-0.24 \pm 0.32,{ }^{12}$ and the standard-model value $v_{l}=-0.07$.

The forward-backward charge asymmetry $A_{\mathrm{FB}}$ for leptons in $e^{+} e^{-}$annihilation is defined as the difference of the integrated differential cross sections for the positively charged lepton emitted into the forward and backward hemispheres, where the forward hemisphere is defined by the direction of the positron beam. For the combined lepton sample of 9 muon and $15 \tau$ events from the region around the $Z$ pole, $90.6 \mathrm{GeV}<E_{\text {c.m. }}<91.5 \mathrm{GeV}$, we measure $A_{\mathrm{FB}}=0.05 \pm 0.22$, where a radiative correction of +0.02 has been applied. ${ }^{13}$ For our data set we expect $A_{\mathrm{FB}}=0.026$ for $M_{Z}=91.14 \mathrm{GeV} / c^{2}$, in good agreement with the measurement, but the result is not precise enough to place useful limits on standard-model parameters.

In conclusion, we have measured the ratios of partial widths of the $Z$ decaying into electrons, muons, and $\tau$ leptons to the hadronic partial width. We have derived the hadronic partial width and values for the vector coupling constants of the $\mu$ and $\tau$ leptons. These measurements confirm within their statistical precision the weak couplings of the leptons and quarks to the $Z$ boson as predicted by the standard model.

One of us (M. Kuhlen) wishes to acknowledge the Alexander von Humboldt Foundation for its support. This work was funded in part by Department of Energy, Contracts No. DE-AC03-81-ER40050 (California Institute of Technology), No. DE-AM03-76SF00010 (University of California, Santa Cruz), No. DE-AC0286ER40253 (University of Colorado), No. DE-AC0383ER40103 (University of Hawaii), No. DE-AC0284ER40125 (Indiana University), No. DE-AC0376SF00098 (LBL), No. DE-AC02-76ER01112 (University of Michigan), and No. DE-AC03-76SF00515 (SLAC), and by the National Science Foundation (Johns Hopkins University).

${ }^{1}$ W. F. L. Hollik, DESY Report No. DESY 88-188, 1988 (unpublished). A top-quark mass of $100 \mathrm{GeV} / c^{2}$ is assumed, and a QCD correction of 5\% has been applied for the hadronic width.

${ }^{2}$ G. S. Abrams et al., Nucl. Instrum. Methods Phys. Res., Sect. A 281, 55 (1989).

${ }^{3}$ T. Sjöstrand, Comput. Phys. Commun. 39, 347 (1986). This model was found to describe well the general featues of hadronic $Z$ decays; see G. S. Abrams et al., Phys. Rev. Lett. 63, 1558 (1989).

${ }^{4}$ S. Jadach and Z. Was, Comput. Phys. Commun. 36, 197 
(1985).

${ }^{5}$ F. A. Berends, W. Hollik, and R. Kleiss, Nucl. Phys. B304, 712 (1988).

${ }^{6}$ F. A. Berends, P. H. Daverveldt, and R. Kleiss, Comput. Phys. Commun. 40, 309 (1986).

${ }^{7}$ The statistical errors are calculated as $68.3 \%$-C.L. intervals using the technique of F. James and M. Roos, Nucl. Phys. B172, 475 (1980).

${ }^{8}$ The overall detection efficiencies for hadronic, electron, muon, and $\tau$ events are $0.88 \pm 0.04,0.62 \pm 0.04,0.54 \pm 0.03$, and $0.66 \pm 0.04$, respectively. The corrected numbers of events are $450 \pm 30$ hadronic events, $16.7 \pm 5.6 e$ events $23.7 \pm 6.8 \mu$ events, and $29.5 \pm 7.2 \tau$ events.

${ }^{9}$ R. N. Cahn, Phys. Rev. D 36, 2666 (1987).

${ }^{10}$ G. S. Abrams et al., Phys. Rev. Lett. 63, 2173 (1989).

${ }^{11}$ W. T. Ford et al., Phys. Rev. D 36, 1971 (1987).

${ }^{12}$ A. Argento et al., Phys. Lett. 120B, 245 (1983). This measurement assumes standard-model couplings for the quarks.

${ }^{13}$ J. E. Campagne and R. Zitoun, Phys. Lett. B 222, 497 (1989). 